\title{
La importancia del teléfono móvil para la comunicación publicitaria
}

\author{
José Ignacio NiÑo GonZÁLEZ \\ Universidad Complutense de Madrid \\ josenino@ccinf.ucm.es
}

\begin{abstract}
Resumen
La publicidad, en su constante búsqueda de vías alternativas para evitar los saturados medios tradicionales, no puede perder de vista las posibilidades que le ofrece el teléfono móvil como medio de comunicación persuasiva. Con objeto de obtener el máximo rendimiento de estas innegables condiciones, hay que conocer cuáles son las líneas de explotación publicitaria que ofrecen mejores opciones para desarrollar acciones eficaces y entender cómo se ejecutan.
\end{abstract}

Palabras claves: Teléfono móvil, publicidad, tecnología, comunicación

\section{The importance of mobile phone advertising}

\begin{abstract}
Advertising, in its constant search for alternative ways to avoid saturated traditional media cannot lose sight of the possibilities offered by mobile phone as a means of persuasive communication. In order to get the most out of these undeniable conditions, we must know which advertising business lines offer better options to develop effective actions and understand how they run.
\end{abstract}

Key Words: Mobile phone, advertising, technology, communication

\section{Referencia normalizada:}

Niño González, J. I. (2013) La importancia del teléfono móvil para la comunicación publicitaria. Historia y Comunicación Social. Vol. 18 Nº Especial Octubre. Págs. 581-590.

Sumario: 1. Introducción. 2. Metodología. 3. Análisis e interpretación de datos. 4. Conclusiones. 5. Bibliografía.

\section{Introducción}

En las últimas décadas la sociedad ha experimentado una profunda transformación que se fundamenta en el desarrollo tecnológico y que tiene una especial significación en el ámbito de la comunicación. Para Duderstadt (2002) el cambio en la forma en la que los individuos se interrelacionan con el entorno le lleva a afirmar que la sociedad está experimentando una evolución que es el origen de la Sociedad de la Información. Dicha transformación alcanza su punto culminante con la irrupción de la tecnología inalámbrica, un avance científico que ha modificado aspectos fundamentales del 
comportamiento social. La conocida como "revolución móvil", rompe los estándares vigentes y propicia un nuevo modo de interrelación basado en la movilidad que permite al ser humano estar conectado en cualquier lugar. Estamos en presencia de un cambio de escenario que tiene un protagonista esencial: el teléfono móvil. Hablamos de un dispositivo con una presencia tan fuerte en nuestra vida cotidiana que sorprende por la velocidad con la que se ha introducido de forma tan categórica en la sociedad:

Gráfico 1: fuentes $C M T$ e $I N E$

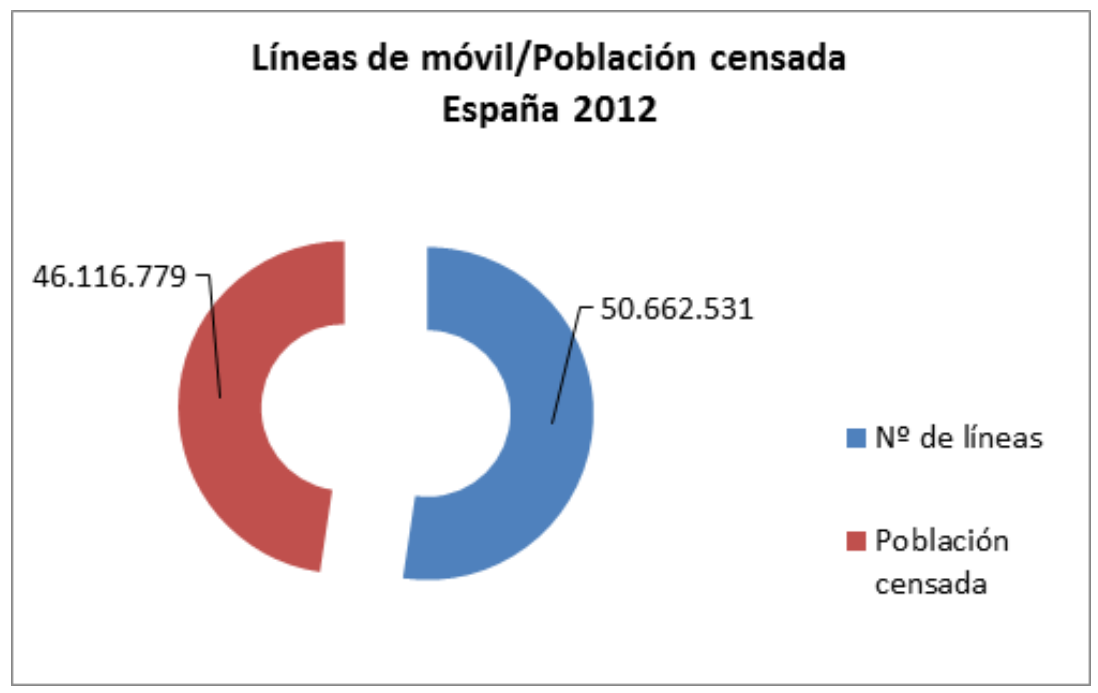

En el grafico 1 podemos observar que el informe elaborado por la CMT (Comisión del Mercado de las Telecomunicaciones) registra 50.662.531 de líneas de teléfonos móviles en España durante el 2012, número que supera con creces a la población censada, que de acuerdo con la información suministrada por el INE (Instituto Nacional de Estadística) para el mismo periodo se cifraba en 46.116 .779 de individuos. Las cifras son contundentes y exponen el elevado grado de penetración del teléfono móvil, pero debemos ir más allá del mero dato numérico para entender cuáles son los factores que dimensionan esta realidad. En el marco de las sociedades modernas, cuanto mayor es el nivel de información, de conocimiento y de aprendizaje, mayor es su capacidad para desarrollar el grado de bienestar. Si en consecuencia con estos requerimientos, las herramientas e instrumentos que resuelven estas necesidades se desarrollan tecnológicamente, es fácil comprender el elevado nivel de aceptación que estos tendrán.

"La tecnología de las comunicaciones, e Internet a la cabeza, es un bien para el hombre y para la humanidad entera; es un elemento de progreso y de cultura, de enriquecedor intercambio y de universal comunicación.” (Méndiz, 2002: 14). 
El impacto de la tecnología en nuestra sociedad de la información es muy grande y cada vez tiene más presencia en nuestros usos cotidianos. En su "Teoría de la Aceptación de la Tecnología" Davis, F. \& Venkatesh, V. (1989) explican que cualquier nueva tecnología supone un reto para el ser humano y por lo tanto puede provocar un rechazo, pero si el individuo entiende que el esfuerzo que le va a llevar familiarizarse con esa tecnología viene justificado por una recompensa en forma de beneficios que le faciliten la vida, no tardará en aceptar esa novedad. En la misma línea, Schiffman y Kanuk (1997) establecen cinco factores que influyen sobre el grado de aceptación de nuevos productos:

- La ventaja relativa: el individuo compara el nuevo producto con los existentes para descubrir que ventajas aporta la novedad.

- La compatibilidad: si el producto es coherente con las necesidades actuales.

- La complejidad de su uso: cuando el individuo entiende que un nuevo producto es fácil de usar se produce una adopción inmediata. Sin embargo, aunque el individuo perciba que es un producto que le ofrece muchas ventajas, si se ve incapaz de usarlo con eficacia, lo rechazará.

- La facilidad para ser probado: si el nuevo producto ha podido ser probado con anterioridad.

- La observabilidad: cuando las ventajas del nuevo producto se observan de forma evidente.

Como hemos visto anteriormente, podemos concluir que en España todo el mundo tiene teléfono móvil (exceptuando tramos de edades muy jóvenes y personas de mucha edad) y sin duda es un claro ejemplo de instrumento tecnológico cuyo uso se adopta de forma inmediata porque los usuarios lo perciben como un producto novedoso que aporta grandes ventajas. Estamos ante un dispositivo personal, único y portátil que está con nosotros en cualquier momento y que utilizamos de modo habitual porque nos ayuda a hacernos más fácil muchas tareas de nuestra vida cotidiana.

Pero el terminal móvil no es tan solo una herramienta de comunicación con muchas funcionalidades, también es un dispositivo con incuestionables capacidades mediáticas. Y es en esta sentido, en su condición de medio de comunicación, en el que la publicidad tiene que trabajar para explotar sus posibilidades. Como sistema de comunicación persuasiva, la publicidad tiene que crear vínculos con cualquier medio innovador que le ofrezca la opción de llevar con eficacia mensajes publicitarios a potenciales grandes audiencias. Y esta relación es aún más importante si pensamos que hablamos de un medio que se fundamenta en una tecnología de última generación que tiene una presencia especialmente grande en el segmento formado por los "nativos digitales", pilares básicos de la sociedad digital.

Por tanto, para la publicidad es esencial identificar qué puede aportarle este nuevo medio tecnológico, y así poder realizar acciones de comunicación que aprovechan al máximo todo su potencial. 


\section{Metodología}

En este trabajo el objeto de estudio se analiza desde una perspectiva cualitativa, realizándose entrevistas en profundidad a un grupo de individuos seleccionados por su experiencia y relación con el ámbito de referencia con el fin de recoger y valorar sus aportaciones.

Mediante esta técnica se persigue obtener datos relevantes cuantificados que sirvan al investigador para aislar conceptos y poder establecer un esquema que dé respuestas a las cuestiones planteadas. Es un proceso de análisis no matemático que se basa en el rigor de la cuantificación de las respuestas obtenidas y en la interpretación objetiva por parte del investigador.

"Con el término "investigación cualitativa", entendemos cualquier tipo de investigación que produce hallazgos a los que no se llega por medio de procedimientos estadísticos u otros medios de cuantificación" (Strauss \& Corbin, 2002: 11).

Las entrevistas en profundidad se realizan sobre un colectivo de expertos con un perfil profesional que en conjunto cubre todas las áreas de actuación en las que se desarrolla la actividad publicitaria en el dispositivo móvil: anunciante, publicitario, experto en medios, experto en contenidos, consultor tecnológico y empresa de tecnología.

La entrevista comienza con unas preguntas que buscan centrar el objeto de estudio y establecer un hilo conductor que sirva para conocer en detalle las opiniones de los entrevistados tanto desde el plano personal como profesional.

2.1 Perfil de los entrevistados

\begin{tabular}{|l|l|}
\hline Perfil & Empresa \\
\hline Director General & Glodimark \\
\hline Director de Marketing & Sennheiser \\
\hline Director Quality Procedures & Barclays Bank \\
\hline Consejero Delegado & ZenithOptimedia \\
\hline Director de Operaciones & Amper \\
\hline Director General & Novavoz \\
\hline Director de Innovación & Ba-aba Software \\
\hline Director de Nuevos negocios & Presentalia \\
\hline Profesor & Universidad Rey Juan Carlos \\
\hline
\end{tabular}




\subsection{Cuestionario de la entrevista}

Las preguntas propuestas como hilo conductor son:

$1 /$ ¿Qué aportan y qué no aportan las nuevas tecnologías a la publicidad?

2/ ¿Tiene proyección de futuro la publicidad en el móvil y por qué?

3/ ¿Cómo se relaciona el móvil en su vertiente publicitaria con las redes sociales?

\subsection{Objeto formal}

Este trabajo realiza un análisis que busca determinar cuáles son para los diferentes profesionales las principales características que entienden aporta el teléfono móvil a la actividad publicitaria y como se deben aplicar estas innovaciones con objeto de conseguir campañas cada vez más eficaces y rentables.

2.4 Preguntas de investigación

En esta investigación las preguntas fundamentales son:

¿Qué aportan las nuevas tecnologías a la actividad publicitaria en el teléfono móvil?

¿Qué piensan de la combinación publicidad-redes sociales en el teléfono móvil?

\subsection{Objetivos}

Identificar que variables diferenciadoras aportan las nuevas tecnologías a la publicidad.

Establecer que contenidos publicitarios pueden ser los que funcionan en el ámbito de las redes sociales a través del terminal móvil.

\subsection{Hipótesis}

Hipótesis particular 1: El teléfono móvil es un medio con alto nivel de interactividad que permite medir las acciones publicitarias con precisión.

Hipótesis particular 2: La inversión en publicidad en redes sociales se va a desarrollar con especial intensidad en el ámbito de los dispositivos móviles.

Hipótesis nuclear: El teléfono móvil posee características que le permiten postularse como medio de gran potencial para la publicidad. 


\section{Análisis e interpretación de datos}

Se realiza el análisis considerando 4 niveles desde diferentes planos de investigación: horizontal, transversal, relacional y global. El plano horizontal busca identificar en cada pregunta los conceptos relevantes. El análisis transversal establece la interrelación de estos conceptos entre todas las preguntas. El plano relacional identifica y relaciona los conceptos que se han expresado de forma diferente pero con una misma intención de significado. El nivel de análisis global da una perspectiva integrada de los resultados obtenidos en los anteriores niveles.

\subsection{Análisis horizontal, transversal y relacional}

La primera cuestión planteada al grupo de entrevistados persigue conocer cuál es en su opinión la principal aportación de esta nueva tecnología a la publicidad y nos encontramos con un concepto dominante: hay una coincidencia generalizada en que el aspecto básico que aporta el teléfono móvil es la gran capacidad que tiene este medio para medir los resultados de las acciones publicitarias que se realizan en él. Para la actividad publicitaria la medición es esencial máxime si entendemos que en la actualidad las campañas se articulan en torno a un parámetro básico: el retorno de la inversión de las mismas. En este sentido es básico disponer de medios que permitan medir con exactitud los resultados obtenidos.

También vemos que aparecen dos conceptos con intensidad. En primer lugar para los entrevistados el teléfono móvil permite que los receptores sean creadores de contenido. Por tanto, si se conoce que tipos de contenidos genera cada usuario, la publicidad estará en disposición de establecer un perfil de ese individuo e impactarle con contenidos persuasivos muy afines con sus gustos y necesidades. En segundo lugar nuestro panel de expertos señala la interactividad como otra de las grandes aportaciones de la telefonía móvil para la actividad publicitaria. Gracias a esta condición, la relación con el receptor se convierte en un dialogo que permite identificar con claridad sus necesidades y en consecuencia la publicidad puede actuar con mensajes muy eficaces. En el siguiente gráfico podemos ver como se concretan numéricamente las expresiones de conceptos realizadas por los entrevistados:

Respecto a la segunda pregunta planteada, los entrevistados coinciden al señalar que las empresas son conscientes de la proyección de futuro de esta publicidad, sin embargo matizan que es una actividad que está todavía en fase de desarrollo pero en la que hay que estar ya que los próximos años vendrán marcados por una predominancia de la actividad publicitaria digital y de un perfil de potencial consumidor que será un "nativo digital".

Con la tercera pregunta los entrevistados opinan sobre cómo ven la publicidad en las redes sociales y la valoración es muy positiva. Establecen un paralelismo con el auge que están experimentando las redes sociales a través del ordenador personal y consideran que si en este dispositivo las perspectivas son muy atractivas, más potencial existe en un dispositivo que reúne todas las características del ordenador pero que 
además suma su portabilidad y capacidad para conectarse a las redes sociales desde cualquier lugar en cualquier momento.

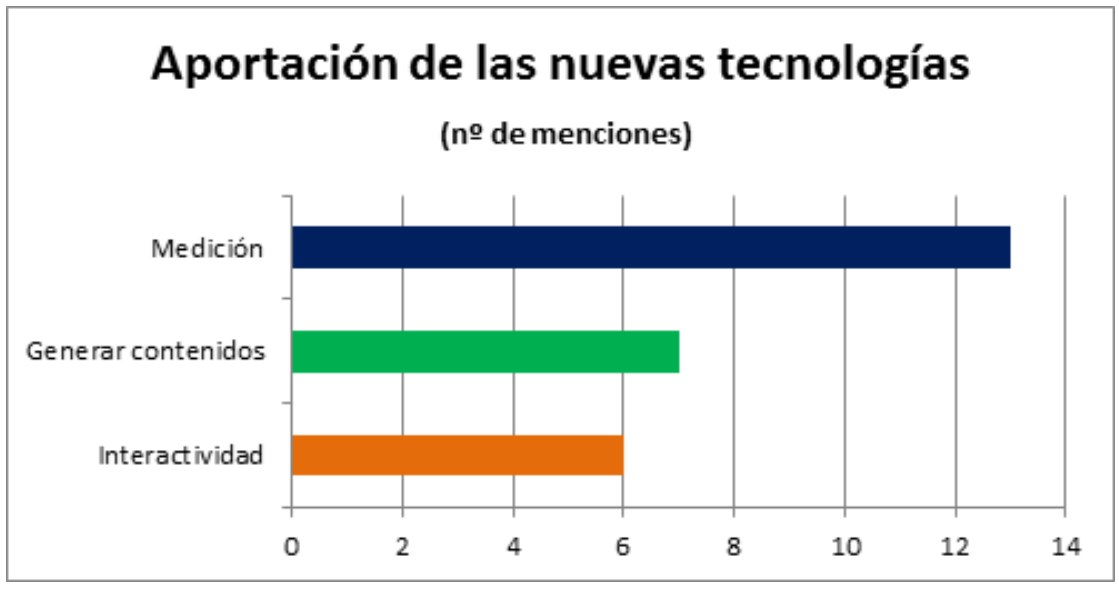

Fuente: Propia.

Las posibilidades que ofrecen las redes sociales para la publicidad generan un gran número de comentarios como se constata en el gráfico que observamos a continuación:

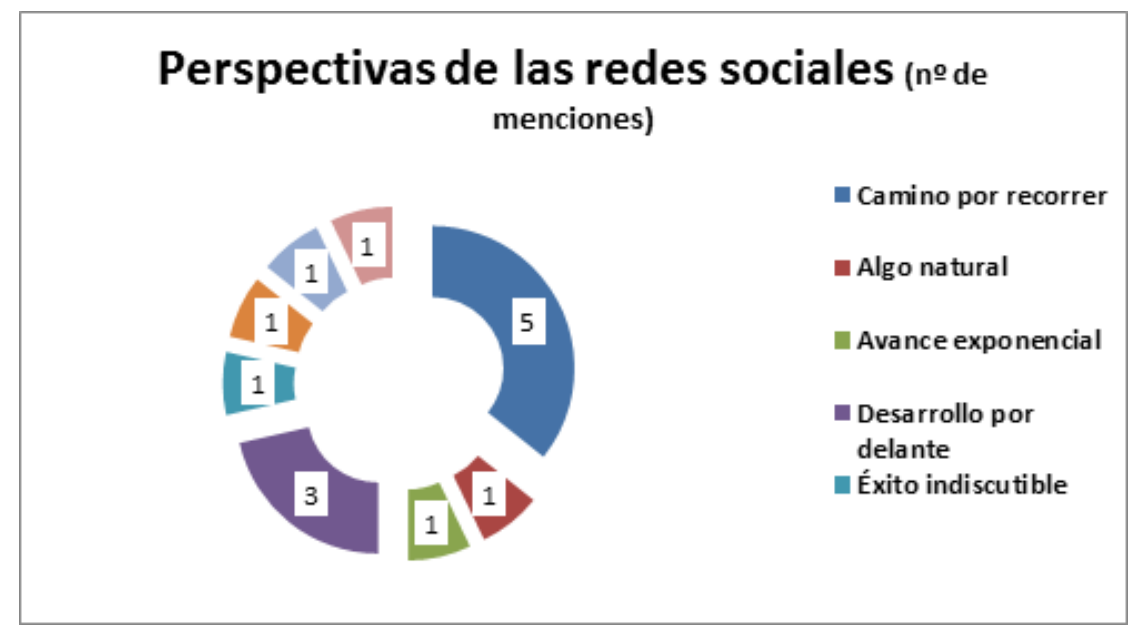

Fuente: Propia.

\subsection{Análisis global e interpretación de datos}

Los expertos entrevistados consideran que la publicidad tiene en el terminal móvil un medio con unas posibilidades de desarrollo innovadoras, hasta ahora ningún medio podía ofrecer a la comunicación publicitaria las opciones que pone a su alcance la 
telefonía móvil. Como aportación destacada aparece la capacidad de medición que ofrece este tipo de publicidad, una prestación presente en todas las modalidades de publicidad basada en las nuevas tecnologías pero que tiene especial relevancia en el teléfono móvil. Las acciones se pueden medir con mucho rigor y los perfiles de los potenciales usuarios están identificados.

\section{Conclusiones}

Los expertos coinciden en que las nuevas tecnologías dotan a la publicidad de capacidades únicas como es la interactividad o identificación de perfiles de usuarios, pero por encima destaca la capacidad de medición, la de mayor rigor conocida hasta por la publicidad. Y cuanto más cerca está el entrevistado de la planificación de medios, más relevante es esta afirmación ya que es donde esta capacidad tiene más significación y representa una de las variables más importantes en esta actividad.

Por tanto podemos decir que la hipótesis particular 1 se cumple y que la publicidad en el móvil es una actividad que se puede medir con gran rigor y que el teléfono móvil está dotado de una gran capacidad interactiva.

En cuanto a la segunda cuestión en estudio, los entrevistados no tienen dudas e identifican la publicidad en redes sociales a través del móvil como un campo de gran desarrollo y enorme potencial. De hecho, los expertos explican que en sus empresas se están destinando partidas presupuestarias para desarrollar acciones en redes sociales y que la tendencia es incrementar los esfuerzos en éste área aun viviéndose momentos de reducción de inversión en el resto de actividades publicitarias. También reflejan como dato que apoya estas opiniones el que muchos fabricantes de terminales móviles ofrezcan aparatos desarrollados específicamente para interactuar con las redes sociales en movilidad. Este aspecto, unido a la mejora de contenidos de la publicidad desplegada en las redes sociales dan muestra del gran futuro que espera a esta forma de hacer comunicación persuasiva.

Con estas afirmaciones, podemos concluir que la hipótesis particular 2 se confirma y afirmar que en las redes sociales a través del teléfono móvil la publicidad tiene un campo de desarrollo de gran potencial.

Por tanto, la confirmación de las hipótesis particulares permiten afirmar que el teléfono móvil aporta a la publicidad condiciones esenciales para ser un medio esencial para desarrollar campañas publicitarias en los próximos años y por tanto verificamos la hipótesis nuclear. 


\section{Bibliografía}

Libros

AGUADO, J.M. \& MARTÍNEZ, I. (2008): “Sociedad móvil: tecnología, identidad y cultura". Biblioteca Nueva. Madrid.

FUNDACIÓN DE LA INNOVACIÓN BANKINTER (2007): “Web 2.0: el negocio de las redes sociales". Madrid.

IAB SPAIN (2012): "Estudio de Inversión en Publicidad Digital". Madrid

ITO, M. \& OKABE, D. \& MATSUDA, M. (2005): "Personal, Portable, Pedestrian: mobile phones in Japanese life". MIT Press. Cambridge (Mass).

MÉNDIZ, A. \& VICTORIA, J. S. (2002): "Publicidad, Comunicación y Marketing en Internet”. Área de Cultura y Educación. Diputación Provincial de Málaga.

ONTSI (2011): "Informe anual de los contenidos digitales en España. Industria y hábitos de consumo". Ministerio de Industria, Turismo y Comercio. Madrid.

ONTSI (2011): “Las redes sociales en internet". Ministerio de Industria, Turismo y Comercio. Madrid.

YOUNG, A. \& AITKEN, L. (2007): "Profitable Marketing Communications: a guide to marketing return on investment". Kogan Page Limited. London.

ZENITHOPTIMEDIA (2012): "Estudio sobre los medios digitales”. Madrid.

Artículos en publicaciones web

CMT (2012): “Informe Anual". http://cmtdata.cmt.es/cmtdata/jsp/inf_anual.jsp?tipo $=1$

COMPUTERWORLD (2013): http://www.computerworld.es/movilidad/gartner-el50-de-las-aplicaciones-moviles-seran-hibridas-en-2016.

GARTNER (2013): http://www.gartner.com/imagesrv/digital-marketing/pdfs/ Recognize-the-Importance-of-Digital-Marketing.pdf

GLOBALWEBINDEX (2013): https://www.globalwebindex.net/social-platforms-gwi-8-update-decline-of-local-social-media-platforms/.

GOYAL, S. (2013): "Advertising on Social Media". Scientific Journal of Pure and Applied Sciences. https://www.sjournals.com/index.php/SJPAS/article/download/447/pdf2(5) 220-223. ISSN 2322-2956.

IAB (Interactive Advertising Bureau) (2013): “Glosario". http://www.iabspain.net/ glosario/

IAB (2013): "IV Estudio anual sobre Redes Sociales". http://www.iabspain.net/ wp-content/uploads/downloads/2013/01/IV-estudio-anual-RRSS_reducida.pdf.

INE (2012): “Anuario Estadístico de España 2012". http://www.ine.es/prodyser/ pubweb/anuario12/anu12 02demog.pdf

ZELDMANN, J. (2006): “Web 3.0”. Published in JavaScript, Business, Industry, Usability 95 Comments. 


\section{El autor}

José Ignacio Niño González es Doctor en Publicidad y Relaciones Públicas por la Universidad Complutense de Madrid 